\title{
OVERCOMING ENVIRONMENTAL DOSE RATE CHANGES IN LUMINESCENCE DATING OF WATERLAIN DEPOSITS
}

\author{
BO LI' ${ }^{1}$, SHENG-HUA LI ${ }^{1}$ and ANN G. WINTLE ${ }^{2}$ \\ ${ }^{I}$ Department of Earth Sciences, The University of Hong Kong, \\ Pokfulam Road, Hong Kong, China \\ ${ }^{2}$ Institute of Geography and Earth Sciences, University of Wales, \\ Aberystwyth SY23 3DB, United Kingdom
}

Received 31 January 2007

Accepted 12 December 2007

\begin{abstract}
This study investigates lacustrine and fluvial sediments on the Sala Us River in the Mu Us Desert in central north China. Significant changes in environmental dose rate in part of the section could be shown to have occurred from measurements of the present day radioactivity and by the age reversal for some samples that had been dated by optically stimulated luminescence (OSL) measurements on quartz. These changes in dose rate can be attributed to recent uptake of radioactive elements found in the sediments; this resulted in significant underestimation of the OSL ages. In this study, the new isochron method using $\mathrm{K}$-feldspar grains has been applied to overcome the effects of changes in dose rate. Calculations are used to show that changes in the environmental dose rate factors, i.e. K, $U$, Th, water content and cosmic ray flux, and disequilibrium in the $U$ and $T h$ decay chains, e.g. radon escape, have a negligible effect on the isochron age. After applying the new isochron method, the effects of changes in dose rate caused by recent uptake of radioactive elements and changes in past water content were effectively overcome and true ages are obtained; this was verified by repeating the luminescence isochron measurements on samples of overlying and underlying sediments.
\end{abstract}

Keywords: optical dating, isochron dating, dose rate, uptake of radioisotopes, K-feldspar.

\section{INTRODUCTION}

Optically stimulated luminescence (OSL) dating has been widely applied to the dating of various archaeological and geological sediments (Aitken, 1998; Wintle, 1997). In this technique, the time since the last moment when the silicate grains making up the sediment were exposed to sunlight can be determined. After burial, the OSL signal grows with time, as a result of exposure to ionizing irradiation emitted by naturally occurring radioisotopes (e.g. $\mathrm{U}, \mathrm{Th}$, and $\mathrm{K}$ ) in the sample itself and its surroundings, and by cosmic rays. As a result, the OSL signals from quartz and feldspar can be used to estimate the radiation dose (equivalent dose $D_{\mathrm{e}}$ ) accumulated after deposition. The age of the sediment is given by the ratio of the equivalent dose to the environmental dose rate which is estimated by measuring the concentrations of radioactive elements in the mineral grains themselves and

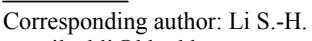

e-mail: shli@hku.hk the surrounding materials.

In the last few years, both the development of new instrumentation and the adoption of a single-aliquot regenerative-dose (SAR) protocol for $D_{\mathrm{e}}$ determination for quartz (Murray and Wintle, 2000) have greatly improved the precision and accuracy of $D_{\mathrm{e}}$ determinations. A similar SAR protocol has been successfully applied to $D_{\mathrm{e}}$ measurement of feldspar (Auclair et al., 2003; Blair et al., 2005; Wallinga et al., 2000; Zhang et al., 2001). However, when compared with $D_{\mathrm{e}}$ determination, little effort has been made to minimize the uncertainty in environmental dose rate estimation, which is equally important for luminescence dating as the $D_{\mathrm{e}}$ measurement.

When determining the environmental dose rate, it is usually assumed that it has remained unchanged since the grains were deposited. However, this will not be the case in geochemically active environments, such as those sediments through which water moves. Changes in dose rate may be caused by several effects, such as disequilibrium in the uranium decay series, e.g. loss of radon, which has been observed in fluvial sediments (Olley et 
al., 1996; Olley et al., 1997; Eriksson et al., 2006), mobility of the radioactive elements (Marley et al., 1993; Mercier et al., 1995), changes of cosmic ray flux and gamma dose rate with burial depth (Aitken, 1985; Munyikwa, 2000) and variation of the water content throughout the burial time causing variable absorption of energy through time in the mineral grains. If one or more of the above factors has changed significantly during the period since deposition, the luminescence ages may be poorly evaluated, even though the $D_{\mathrm{e}}$ values are accurately estimated; this is the result of using present day values of the dose rates in the age calculation.

On the other hand, for alkali feldspar grains a considerable portion of the total dose is derived from the decay of radioactive elements (e.g. ${ }^{40} \mathrm{~K}$ and ${ }^{87} \mathrm{Rb}$ ) that are present in the lattice structure. This means that K-feldspars will receive proportionally less of their total annual dose from their environment than quartz grains of a similar size. The larger the grain, the greater is the contribution from the internal dose. Based on this fact, several methods have been applied to avoid the effect of changes in the environmental dose rate. McKerrell and Mejdahl (1981) proposed a subtraction dating method for thermoluminescence (TL) signals from quartz and alkali feldspar grains of the same size for pottery dating; in their method, the $D_{e}$ of quartz grains is subtracted from that of feldspar grains to obtain the contribution of internal dose within the feldspar grains. This method was later applied to carbonate-cemented coastal sands by Vogel et al. (1999), but the results were considered to be in error (Huntley, 2002) due to the effect of anomalous fading of the infrared stimulated luminescence (IRSL) signal of the feldspar grains. Anomalous fading is commonly observed in both the TL (Wintle, 1973) and optically stimulated luminescence signals (including IRSL) of feldspars (Huntley and Lamothe, 2001). Using TL signals from $\mathrm{K}$-feldspars of different size, as well as TL signals from quartz and plagioclase grains of a fixed size, Mejdahl (1983) went on to propose an isochron dating method in which the measured equivalent dose was plotted as a function of the total beta dose rate. Using such a plot, the age can be derived directly from the slope of the line, and thus it is not necessary to know the environmental gamma dose rate; also the uncertainty associated with the environmental gamma radiation and cosmic ray and external beta radiation is reduced considerably. This approach was not adopted because of the scatter associated with the TL measurements

More recently, a similar method was proposed by Zhao and $\mathrm{Li}$ (2002) in which the isochron was constructed by plotting the measured $D_{\mathrm{e}}$ as a function of grain size. They measured both the OSL signals from quartz grains and IRSL from K-feldspar grains, with different average grain sizes ranging from 108 to $231 \mu \mathrm{m}$. By assuming that the percentage underestimation of $D_{\mathrm{e}}$ for K-feldspars was the same for each of the different grain sizes, an isochron age was obtained directly, without a need for measurement of the environmental dose rate. However, the ages obtained from this method were significantly overestimated when compared with the quartz OSL ages (Zhao and Li, 2002); this was later interpreted by Li et al. (2007) to be the result of assuming the same $D_{\mathrm{e}}$ underestimation factor for K-feldspars of different grain sizes in the natural environment. Li et al. (submitted) proposed a new isochron dating method in which only K-feldspars of different grain sizes were used to obtain values of $D_{e}$. When testing their new method on 13 samples, they found that the new isochron ages were consistent with the quartz OSL ages or ages from geological evidence. This led them to conclude that this method may be used to overcome both anomalous fading of K-feldspars and the effects of past changes in the environmental dose rate.

In this paper, we will report on samples that show evidence for significant changes in environmental dose rate and apply the new isochron method of Li et al. (submitted) to overcome the environmental dose rate changes.

\section{ISOCHRON DATING}

In the isochron dating method (Li et al., submitted), the measured values of $D_{e}$ were plotted against the internal dose rate $\left(\dot{D}_{\text {in }}(s)\right)$ derived using a $\mathrm{K}$ concentration of $13 \pm 1 \%$ (Huntley and Baril, 1997; Zhao and Li, 2005) and $\mathrm{Rb}$ concentration of $400 \pm 100 \mathrm{ppm}$ (Huntley and Hancock, 2001) for each grain size used (Fig. 1). This internal dose rate takes account of the non-infinite size of the grains, such that $D_{i n}(s)=D_{\infty} \Phi_{K}(s)$, where $D_{\infty}$ is the infinite beta dose rate due to ${ }^{40} \mathrm{~K}$ and ${ }^{87} \mathrm{Rb}$ and $\Phi_{K}(s)$ is the combined effective absorption factor for beta particles from $\mathrm{K}$ and $\mathrm{Rb}$ for a grain of size s (Fain et al., 1999). For an infinitely small grain, $\Phi_{k}(s)=0$ and thus $D_{\text {in }}(0)=0$. When the measured values of $D_{e}$ are plotted as a function of the calculated values of $D_{i n}(s)$, they can be projected back onto the dose axis to give $D_{f}(0)$, the $D_{e}$ value for the infinitely small grain only, using linear

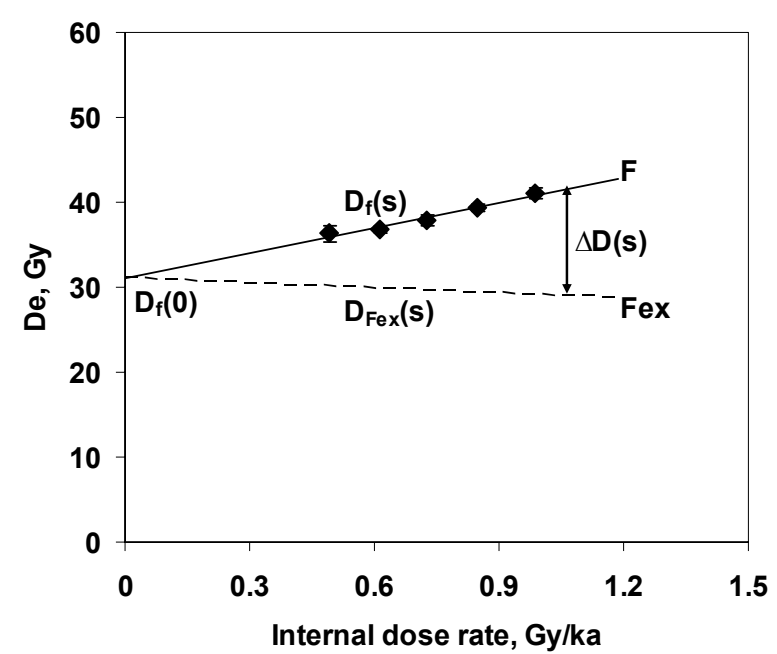

Fig. 1. A typical isochron plot showing the $D_{e}$ values of K-feldspar grains of different sizes plotted against the internal beta dose rate calculated by assuming that the concentrations of $K$ and $R b$ are $13 \%$ and $400 \mu \mathrm{g} / \mathrm{g}$, respectively. The data points are taken from $\mathrm{Li}$ et al. (submitted). Filled symbols are measured data. The full line $F$ is the best fitted line of the data points. The dashed line Fex is calculated using the method described by Li et al. (submitted) and the age calculated from $\Delta D(s)$. 
extrapolation (line F in Fig. 1).

Using the value $D_{f}(0)$ as the effective value for the infinite external dose rate, the contribution of the latter to the grains of different size can be obtained by multiplying $D_{f}(0)$ by the appropriate beta-dose attenuation factor (1- $\Phi)$ for the weighted beta contributions from $\mathrm{U}, \mathrm{Th}, \mathrm{K}$ and $\mathrm{Rb}$ in the bulk sediment (Li et al., 2007). This is shown plotted as the dashed line in Fig 1. Since both this line and the internal dose rates for the data points have been corrected for beta attenuation, the difference between the data points and the dashed line $(\Delta D(s))$ will represent the internal dose received since deposition. If plotting $\Delta D(s)$ against the internal dose rate, the slope would be the age $t$. With simple mathematical calculation, the age $t$ is the slop difference between the trend line $\mathrm{F}$ and the trend line of estimated external dose Fex in Fig. 1.

\section{EFFECTS OF CHANGES IN ENVIRONMENTAL DOSE RATE ON ISOCHRON DATING}

Fig. 1 shows a typical isochron plot (data from Li et al., submitted). Mathematically, the external doses, $D_{f e x}(s)$, for K-feldspar of a specified grain size $(s)$ can be estimated from the equation

$$
\frac{D_{f e x}(s)}{\dot{D}_{e x}(s)}=\frac{D_{f}(0)}{\dot{D}_{e x}(0)}
$$

where $\dot{D}_{e x}(s)$ is the external dose rate for grain size $s$. The value of $\dot{D}_{e x}(s)$ can be estimated based on the environmental $\mathrm{K}, \mathrm{U}$ and Th concentrations, cosmic ray flux and burial depth, and the attenuation factor $(1-\Phi)$ for external beta irradiation (Brennan, 2003; Fain et al., 1999; Mejdahl, 1979). The values of $\Phi$ (the attenuation coefficient) differ slightly for the natural beta flux, depending upon the maximum beta energy of each emitter, e.g. ${ }^{40} \mathrm{~K}$ or individual isotopes in the uranium and thorium decay chains.

Since the calculation of $D_{f e x}(s)$ in line Fex is based on $\dot{D}_{e x}(s)$, which is a function of the relative contributions from environmental $\mathrm{K}, \mathrm{U}$ and $\mathrm{Th}$ concentrations, cosmic rays and water content, it is necessary to investigate how changes in these factors may affect the slope of Fex and thus the isochron age.

The slope of the line Fex in the isochron plot is

$$
S_{F e x}=\frac{D_{f e x}(s)-D_{f}(0)}{\dot{D}_{i n}(s)}=\frac{D_{f}(0)}{\dot{D}_{i n}(s)}\left(\frac{\dot{D}_{e x}(s)}{\dot{D}_{e x}(0)}-1\right)
$$

For a specific grain size $s 1$ and $D_{f}(0), \frac{D_{f}(0)}{\dot{D}_{\text {in }}(s 1)}$ is a constant. The value of $S_{F e x}$ is thus only dependent only on $\left(\frac{\dot{D}_{e x}(s 1)}{\dot{D}_{e x}(0)}-1\right)$, which is a function of the relative contribu- tions of five factors, i.e. the environmental $\mathrm{K}$, $\mathrm{U}$ and $\mathrm{Th}$ concentrations, cosmic ray dose rate and water content. In order to know how each factor may influence $S_{F e x}$, the value of $S_{\mathrm{Fex}}$ was calculated by changing one of the five factors, while keeping the other four factors fixed at typical values, for example, $\mathrm{K}=1.5 \%, \mathrm{U}=3 \mathrm{ppm}, \mathrm{Th}=10$ ppm, no radon loss, water content $=10 \%$ and cosmic ray dose rate $=0.2 \mathrm{~Gy} / \mathrm{ka}$. The normalized slope of Fex $\left(S_{F e x}\right)$ plotted against each of the five factors is shown in Figs $\mathbf{2 a}, \mathbf{b}, \mathbf{c}, \mathbf{d}$, and $\mathbf{e}$, respectively. A summary of the changes in $S_{F e x}$ and isochron age with respect to each of the changes in environmental $\mathrm{K}, \mathrm{U}, \mathrm{Th}$, water content and cosmic ray dose rate is given in Table $\mathbf{1}$. It should be noted that the range of change of each factor is chosen to represent most of the possible sedimentary environments. In reality, the change in each factor will be substantially smaller than the range investigated.

For the change of environmental $\mathrm{K}$ content from $0-3 \%$, the change of $S_{F e x}$ is only within $8 \%$ (Table 1 and the full curve in Fig. 2a). Since the absolute value of $S_{F e x}$ usually contributes less than $20 \%$ of the isochron age ( $\mathrm{Li}$ et al., submitted), such a large variation in $\mathrm{K}$ content can only cause a maximum change of $1.6 \%$ in the isochron age (Table 1). In contrast, such a change in $\mathrm{K}$ content causes the environmental dose rate to change from 1.6 to $4.3 \mathrm{~Gy} / \mathrm{ka}$ (dashed curve in Fig. 2a), which may result in a change in the luminescence age by several hundred percent if a single grain size is used for dating. If the environmental concentration of $U$ changes from 0 to $10 \mathrm{ppm}$, the corresponding value of $S_{\mathrm{Fex}}$ varies by $5 \%$ (full curve in Fig. 2b). This will lead to a change of only $1 \%$ in the isochron age. However, a significant change in the environmental dose rate (up to $200 \%$ ) and the luminescence age is expected for such a change in the environmental $U$ content if a single grain size is used for dating (dashed curve in Fig. 2b). It is to be noted that the loss of radon in the uranium and thorium decay chains may also cause significant change in the dose rate. About $20 \%$ change in the dose rate for a typical composition of radioactive elements in sediment will be resulted from a $100 \%$ loss of radon (Aitken, 1985). However, it is expected to have a maximum of $2 \%$ change in $S_{F e x}$. Therefore, it has negligible effect to the isochron ages.

An even smaller effect on $S_{\mathrm{Fex}}$ (less than 3\%) and the isochron age (less than $0.6 \%$ ) is observed on changing the Th content from 0 to $30 \mathrm{ppm}$ (Fig. 2c), although it has a remarkable influence on the environmental dose rate. Similarly, a change in water content from 0 to $50 \%$ causes a change of less than $4 \%$ in the value of $S_{\mathrm{Fex}}$ and less than $1 \%$ in the isochron age (Fig. 2d). Finally, 10\% variation in $S_{\mathrm{Fex}}$ or $2 \%$ variation in isochron age (Fig. 2e) is expected if the cosmic ray dose rate changes from 0 to $0.5 \mathrm{~Gy} / \mathrm{ka}$, a range that encompasses its value several $\mathrm{km}$ below the Earth's surface near the equator to its value near the surface on the Tibetan Plateau.

If all the variations caused by each of the changes in the five factors $(\mathrm{K}, \mathrm{U}, \mathrm{Th}$, water content and cosmic ray dose rate) are summed in quadrature, the maximum variations in $S_{\mathrm{Fex}}$ and the isochron age are estimated to be $15 \%$ and 3\%, respectively (Table 1). This result suggests that the isochron dating method using K-feldspar grains with different grain sizes has little dependence on changes in 

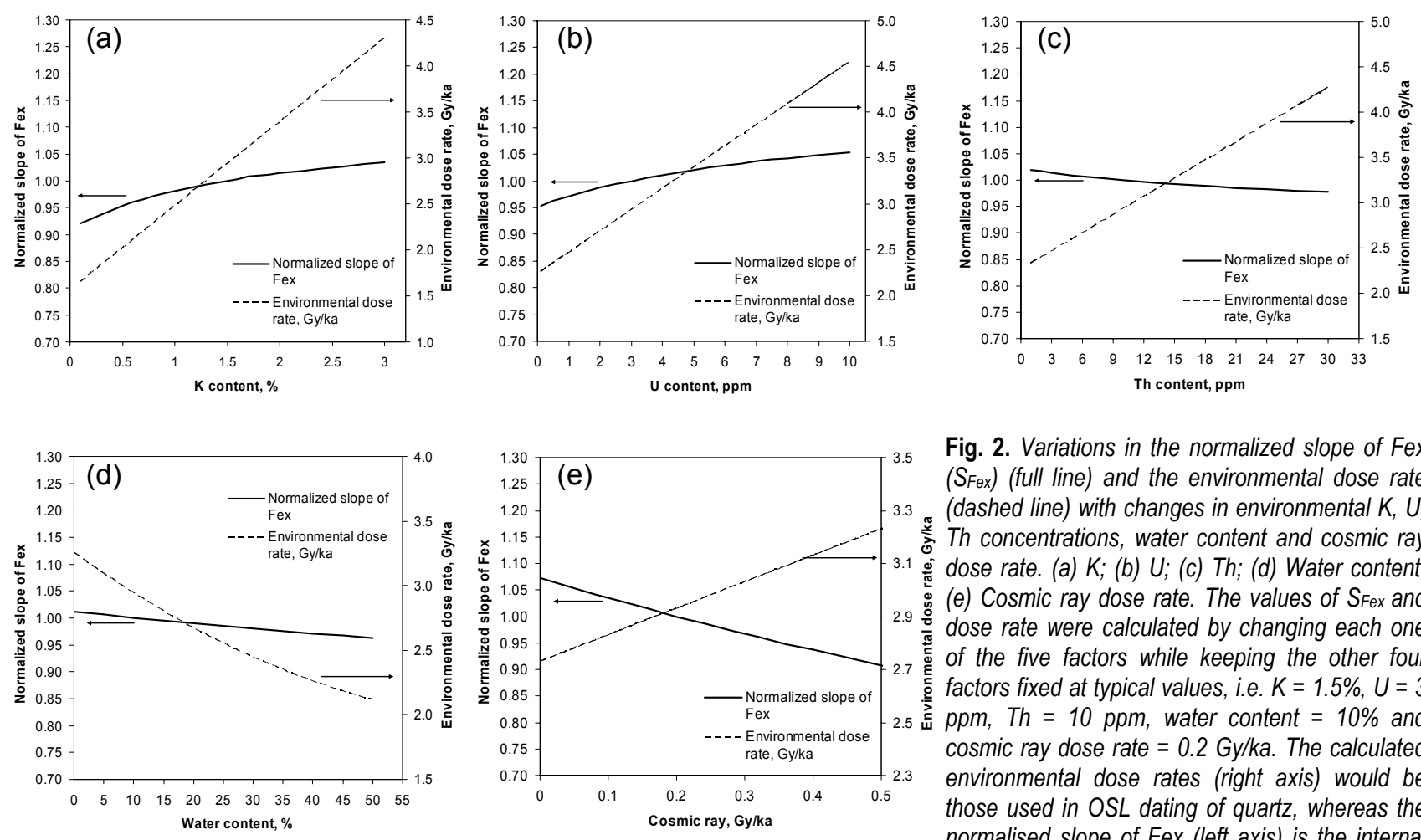

Fig. 2. Variations in the normalized slope of Fex (SFex) (full line) and the environmental dose rate (dashed line) with changes in environmental $\mathrm{K}, \mathrm{U}$, Th concentrations, water content and cosmic ray dose rate. (a) K; (b) U; (c) Th; (d) Water content; (e) Cosmic ray dose rate. The values of $S_{F e x}$ and dose rate were calculated by changing each one of the five factors while keeping the other four factors fixed at typical values, i.e. $K=1.5 \%, U=3$ $\mathrm{ppm}, \mathrm{Th}=10 \mathrm{ppm}$, water content $=10 \%$ and cosmic ray dose rate $=0.2 \mathrm{~Gy} / \mathrm{ka}$. The calculated environmental dose rates (right axis) would be those used in OSL dating of quartz, whereas the normalised slope of Fex (left axis) is the internal dose rate parameter used in the isochron method.

Table 1. Factors related to dose rate that were varied in a theoretical calculation of the isochron age and the percentage effect on the age.

\begin{tabular}{r|ccccc|c}
\hline Factor & $\mathrm{K}$ & $\mathrm{U}$ & $\mathrm{Th}$ & Water content & Cosmic ray & Total \\
\hline Factor range & $0-3 \%$ & $0-10 \mathrm{ppm}$ & $0-30 \mathrm{ppm}$ & $0-50 \%$ & $0-0.5 \mathrm{~Gy} / \mathrm{ka}$ & - \\
\hline Variation of $\boldsymbol{S}_{\mathrm{Fex}}$ & $8 \%$ & $5 \%$ & $3 \%$ & $4 \%$ & $10 \%$ & $15 \%$ \\
\hline Variation of isochron age & $1.6 \%$ & $1.0 \%$ & $0.6 \%$ & $0.8 \%$ & $2.0 \%$ & $3 \%$ \\
\hline
\end{tabular}

the environmental dose rate. Therefore, it is concluded that one can even assume a typical sedimentary environment to calculate the environmental dose rate and the slope of Fex $\left(S_{F e x}\right)$.

\section{SAMPLES AND EXPERIMENTAL APPARATUS}

The samples analysed were Holocene lacustrine and fluvial deposits collected from Dagouwan section along the Sala Us River in the $\mathrm{Mu}$ Us desert area $\left(37^{\circ} 28^{\prime}\right.$ $39^{\circ} 23^{\prime} \mathrm{N}$; $106^{\circ} 10^{\prime}-110^{\circ} 30^{\prime} \mathrm{E}$ ) in central north China (Fig. 3). This area is located at the loess-desert transition belt and thus the sediments will provide enough grains in a range of sizes for an isochron approach to be carried out. The Holocene deposits are two meters thick and are overlying a thick layer of aeolian sands. A detailed description of the stratigraphy of this part of the section has been given by Sun (2000). Grains over $100 \mu \mathrm{m}$ diameter in these lacustrine and fluvial sediments, underlying the modern dune sands, are originally from aeolian sand deposits. The grains have been reworked by water on the bottom of a lake or on the bed of a river and have been incorporated in the resultant lacustrine and fluvial sediments.

Ten samples were collected for luminescence dating (Table 2). They were taken at intervals through a $2.5 \mathrm{~m}$

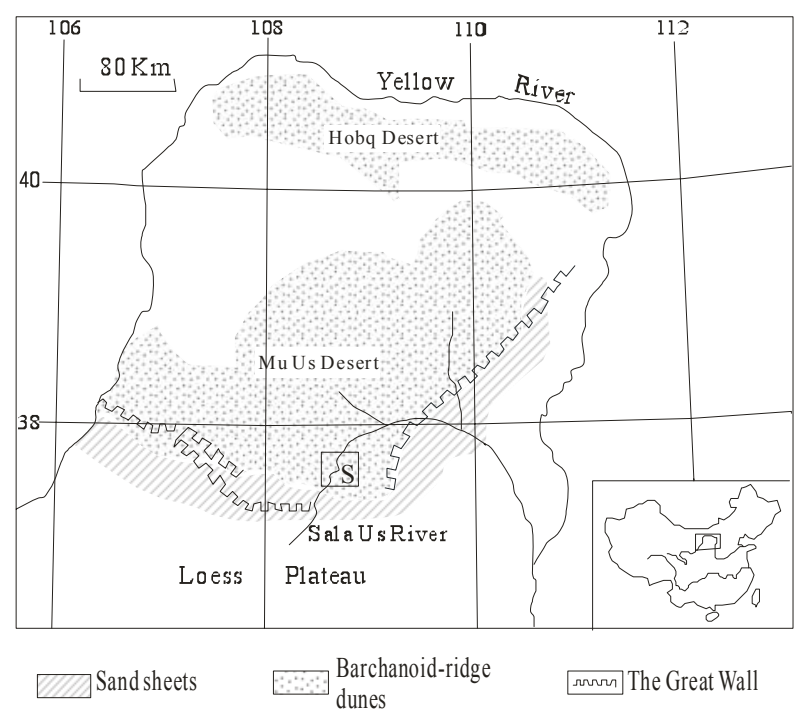

Fig. 3. Map of the Mu Us Desert showing the location of the sampling site (S).

thick section, from the upper part of the underlying aeolian sands (Dgw1) up to the top of the fluvial deposits (Dgw10). All samples were obtained by hammering aluminium cans into freshly cleaned vertical sections or by 
taking block samples. The sample cans and block samples were protected from light immediately after taking them from the section, then sealed inside black plastic bags with tape. In the laboratory, the material at each end of the can and the surface layer of bulk samples was scraped away and used for dose rate measurements. All samples were routinely treated with $\mathrm{HCl}$ and $\mathrm{H}_{2} \mathrm{O}_{2}$ to remove carbonate and organic matter and dried. Different grain size fractions, 63-90, 90-125, 125-150, 150-180, 180-212 and $212-250 \mu \mathrm{m}$, were obtained by dry sieving. At least four grain size fractions were obtained for each sample. Each grain size fraction of the quartz and K-feldspar was then separated using heavy liquids. The separated quartz grains were treated with $40 \% \mathrm{HF}$ for 2 hours to remove any remaining feldspar. For $\mathrm{K}$-feldspars grains, etching was carried out using 10\% HF for 40 minutes. Separated quartz and K-feldspar grains were mounted on $10 \mathrm{~mm}$ diameter aluminium discs with "Silkospay" silicone oil prior to measurement. The purity of the quartz grains was tested by monitoring the infrared bleached OSL signal (Duller, 2003) and measuring the $110^{\circ} \mathrm{C}$ TL peak (Duller, 2003; Li et al., 2002).

All OSL and IRSL measurements were performed on an automated Risø TL-DA-12 reader in the Luminescence Dating Laboratory of the University of Hong Kong. The stimulating light source for K-feldspar is an array of IR emitting diodes $\left(830 \pm 10 \mathrm{~nm} ; \sim 200 \mathrm{~mW} / \mathrm{cm}^{2}\right)$; the stimulating light source for quartz is a tungsten-halogen lamp, the output of which is filtered to provide light in the region from 420 to $550 \mathrm{~nm}$ using a Schott GG-420 filter combined with interference filters to provide stimulation power of $\sim 40 \mathrm{~mW} / \mathrm{cm}^{2}$. The OSL signals from quartz were detected through two $3 \mathrm{~mm}$ thick U-340 filters. The IRSL signals from feldspar were detected through a Schott BG-39 filter combined with a Corning 7-59 filter. Irradiation was carried out using a ${ }^{90} \mathrm{Sr} /{ }^{90} \mathrm{Y}$ beta source with a dose rate of $0.042 \mathrm{~Gy} / \mathrm{s}$ to quartz grains of $125-150 \mu \mathrm{m}$ in diameter. For IRSL measurements of K-feldspars, the relative dose rate to different grain sizes for the ${ }^{90} \mathrm{Sr} /{ }^{90} \mathrm{Y}$ beta source was obtained using quartz of different grain sizes extracted from the same sediments; the quartz had been heated and then given a known gamma dose (see Li et al., 2007).

\section{RESULTS}

\section{Optical dating using quartz and problems with annual dose}

The $D_{\mathrm{e}}$ values of quartz were measured using a singlealiquot regenerative-dose (SAR) protocol, following the procedure of Murray and Wintle (2000) with preheating at $260^{\circ} \mathrm{C}$ for $10 \mathrm{~s}$ and a cut-heat at $220^{\circ} \mathrm{C}$ after each regenerative dose and the test dose, respectively. Five regenerative doses, including one zero dose and one repeat dose, were applied to each disc. The OSL decay curve was measured by holding the sample at $125^{\circ} \mathrm{C}$ for $100 \mathrm{~s}$. The OSL signals, $L_{n}$ and $L_{x}$, used for $D_{\mathrm{e}}$ calculation were obtained by integrating the OSL counts in the first $2 \mathrm{~s}$ and subtracting an equivalent signal taken from the last $10 \mathrm{~s}$. The sensitivity changes throughout the measurement sequence were monitored by measuring the test dose OSL, $T_{n}$ and $T_{x}$. The $D_{\mathrm{e}}$ values were then estimated using the corrected OSL intensities $L_{n} / T_{n}$ and $L_{x} / T_{x}$. Aliquots with a recycling ratio falling outside the range of $1.0 \pm 0.1$ were discarded for age calculation.

The environmental dose rate was measured using a variety of techniques. Thick source alpha counting (TSAC) was used on dried samples to measure the radioactivity of the decay chains of $U$ and Th. The alpha count rates were converted into alpha, beta and gamma dose rates according to the conversion factors given by Adamiec and Aitken (1998). The K content was measured using X-Ray Fluorescence (XRF) spectroscopy. The cosmic ray dose rate was calculated from the burial depth and the geographic position (altitude, longitude and latitude) of the sampling site (Prescott and Hutton, 1994). Since the samples were initially saturated with water but then dried out after the disappearance of the lake and river in the last $2 \mathrm{ka}$ (as deduced by the age of the uppermost sample), measurement of the present day water content does not represent the average water content over time since deposition. The water content of the samples was estimated by measuring the saturated water content of the samples in the laboratory. The saturated water content was found to be $\sim 25 \%$. Therefore, a value of $20 \pm 5 \%$ was chosen as the average water content, after allowing for the samples to be dry for the last $2 \mathrm{ka}$.

A summary of the $D_{\mathrm{e}}$ values, environmental dose rates and the OSL ages for the quartz grains are listed in Table 2. The quartz ages show inconsistency with the

Table 2. Sample number, depth below top of fluvial unit, phases and information relating to OSL dating of the quartz (125-150 $\mu \mathrm{m})$ fraction.

\begin{tabular}{lllccccccc}
\hline Sample & $\begin{array}{c}\text { Depth } \\
(\mathbf{m})\end{array}$ & Phase & $\begin{array}{c}\mathbf{K} \\
(\%)\end{array}$ & $\begin{array}{c}\boldsymbol{\alpha} \text {-counting } \\
(\mathbf{c} / \mathbf{k s})^{\mathbf{a}}\end{array}$ & $\begin{array}{c}\text { Water } \\
\text { Content (\%) }\end{array}$ & $\begin{array}{c}\text { Cosmic ray } \\
(\mathbf{G y} / \mathbf{k a})\end{array}$ & $\begin{array}{c}\text { De(Q) } \\
(\mathbf{G y})\end{array}$ & $\begin{array}{c}\text { Dose rate } \\
(\mathbf{G y} / \mathbf{k a})\end{array}$ & $\begin{array}{c}\text { Age } \\
(\mathbf{k a})\end{array}$ \\
\hline Dgw10 & 0.05 & Fluvial & $1.36 \pm 0.04$ & 8.31 & $20 \pm 5$ & 0.235 & $5.15 \pm 0.20$ & $2.31 \pm 0.08$ & $2.19 \pm 0.09$ \\
Dgw9 & 0.6 & Fluvial & $1.04 \pm 0.03$ & 5.99 & $20 \pm 5$ & 0.218 & $8.92 \pm 0.45$ & $1.76 \pm 0.07$ & $5.04 \pm 0.30$ \\
Dgw8 & 0.7 & Lacustrine & $1.85 \pm 0.06$ & 6.28 & $20 \pm 5$ & 0.212 & $17.33 \pm 0.64$ & $2.43 \pm 0.08$ & $7.06 \pm 0.27$ \\
Dgw7 & 1 & Lacustrine & $1.81 \pm 0.05$ & 8.30 & $20 \pm 5$ & 0.207 & $18.33 \pm 0.79$ & $2.63 \pm 0.10$ & $6.91 \pm 0.32$ \\
Dgw6 & 1.15 & Lacustrine & $1.93 \pm 0.06$ & 7.80 & $20 \pm 5$ & 0.204 & $21.14 \pm 0.61$ & $2.67 \pm 0.10$ & $7.85 \pm 0.25$ \\
Dgw5 & 1.35 & Lacustrine & $1.94 \pm 0.06$ & 7.30 & $20 \pm 5$ & 0.198 & $21.73 \pm 0.67$ & $2.61 \pm 0.10$ & $8.25 \pm 0.29$ \\
Dgw4 & 1.55 & Lacustrine & $2.18 \pm 0.07$ & 12.84 & $20 \pm 5$ & 0.193 & $22.68 \pm 0.68$ & $3.46 \pm 0.13$ & $6.51 \pm 0.21$ \\
Dgw3 & 1.75 & Lacustrine & $1.96 \pm 0.06$ & 27.26 & $20 \pm 5$ & 0.186 & $24.07 \pm 0.81$ & $5.01 \pm 0.19$ & $4.78 \pm 0.16$ \\
Dgw2 & 1.95 & Lacustrine & $1.97 \pm 0.06$ & 18.77 & $20 \pm 5$ & 0.181 & $27.46 \pm 0.84$ & $4.00 \pm 0.15$ & $6.84 \pm 0.21$ \\
Dgw1 & 2.5 & Eolian & $1.83 \pm 0.05$ & 6.13 & $15 \pm 5$ & 0.169 & $20.67 \pm 0.69$ & $2.46 \pm 0.10$ & $8.35 \pm 0.32$ \\
\hline
\end{tabular}

a The a-count rate is measured using $42-\mathrm{mm}$-diameter ZnS screens. 
stratigraphy. From sample Dgw5 to Dgw10, the ages decrease monotonically from 7.85 to $2.19 \mathrm{ka}$. However, the ages for the lacustrine samples (Dgw2, Dgw3 and Dgw4) $6.84,4.78$ and $6.51 \mathrm{ka}$, respectively, are significantly smaller than the ages for the four overlying samples (Dgw5 to Dgw8). This age reversal cannot be due to the incomplete bleaching of the quartz grains of these samples because the grains in these sediments were originally derived from eolian sand and have been reworked by water either at the bottom of a shallow lake or small river. The grains had been well bleached before deposition as checked with luminescence techniques ( $\mathrm{Li}, 1994$; $\mathrm{Li}, 2001)$. It is also unlikely that the age reversal is a result of incorrect estimation of $D_{\mathrm{e}}$ values since the $D_{\mathrm{e}}$ values for all samples increase monotonically with burial depth from sample Dgw2 to Dgw10 and no reversal is observed (Table 2). Therefore, the explanation for the OSL age reversal for samples Dgw2, Dgw3 and Dgw4 is that their environmental dose rates were incorrectly determined.

There is no noticeable difference between the $\mathrm{K}$ contents for samples Dgw2, Dgw3 and Dgw4 and for those samples above and below them. However, the alpha count rates for samples Dgw2 (12.84 counts/ks), Dgw3 (27.26 counts/ks) and Dgw4 (18.77 counts/ks), especially for Dgw3, are abnormally high when compared to other samples, which are all within the range of 6-9 counts $/ \mathrm{ks}$. This results in calculated dose rates for samples Dgw2, Dgw3 and Dgw4 of 3.46, 5.01 and $4.00 \mathrm{~Gy} / \mathrm{ka}$, respectively, compared with those for the other samples ( $2.6 \mathrm{~Gy} / \mathrm{ka})$, except for sample Dgw9 which is somewhat lower. Therefore, we conclude that the activities of the decay chains of $U$ and $T h$ (as derived from alpha counting) for samples Dgw2, Dgw3 and Dgw4 have changed significantly; thus the dose rates measured today do not represent the effective dose rates that have been experienced by the grains since they were deposited.

\section{Isochron dating using K-feldspar grains}

In order to test that whether or not the age reversal shown above is due to changes in the environmental dose rate, isochron dating using $\mathrm{K}$-feldspar grains was applied to two of the problematic samples, Dgw3 and Dgw4. In addition, sample Dgw5, which is assumed to have no problem with its dose rate, was also measured using isochron dating to confirm that the measured dose rate is correct. The K-feldspar grains of different grain sizes from these samples were measured using a SAR protocol similar to that used for quartz, following the procedures given by Auclair et al. (2003), Huot and Lamothe (2003) and Blair et al. (2005). In this protocol, the same preheat conditions $\left(10 \mathrm{~s}\right.$ preheat at $280^{\circ} \mathrm{C}$ in this study) were used ahead of both the main IRSL measurement (natural and regenerative dose) and the test dose IRSL measurement. The IRSL decay curve was measured at $60^{\circ} \mathrm{C}$ for $400 \mathrm{~s}$. The suitability of this protocol for our samples was tested by application of a dose recovery test and a preheat plateau test (Li et al., 2007).

The results of isochron dating using different grain sizes for samples Dgw3, Dgw4 and Dgw5 are shown in Fig. 4a, b and c, respectively. The internal dose rates for the K-feldspar grains are calculated by assuming values of $13 \pm 1 \%$ and $400 \pm 100 \mathrm{ppm}$ for potassium and rubidium, respectively, as recommended by Huntley and Baril (1997), Zhao and Li (2005) and Huntley and Hancock (2001). Li et al. (submitted) have compared the isochron ages obtained by using in turn values of $11 \%, 13 \%$ and $14 \%$ for the potassium content, they concluded that assuming $13 \pm 1 \%$ for the internal potassium content was appropriate.

The isochron ages, together with the feldspar IRSL and quartz OSL ages for a single grain size $(125-150 \mu \mathrm{m})$, are shown in Table 3. Isochron dating gave ages of $8.3 \pm 1.1 \mathrm{ka}$ and $8.5 \pm 1.0 \mathrm{ka}$ for samples Dgw3 and Dgw4, respectively; these values are consistent with the expected age of $8.3 \pm 0.3 \mathrm{ka}$ deduced from the quartz OSL ages from the underlying sample Dgw1 $(8.35 \pm 0.32 \mathrm{ka})$ and the overlying sample Dgw5 $(8.25 \pm 0.29 \mathrm{ka})$. This result suggests that the quartz OSL ages of Dgw2, Dgw3 and Dgw4 (6.51, 4.78 and $6.51 \mathrm{ka}$, respectively) underestimate the true ages as a result of the changes in the environmental dose rate. On the other hand, the isochron age of Dgw5 is $8.6 \pm 1.5 \mathrm{ka}$, in excellent agreement with the quartz OSL age $8.25 \pm 0.29 \mathrm{ka}$, suggesting that its measured dose rate is reliable. Since the alpha count rates for all samples including Dgw5 are similar, except Dgw2, Dgw3 and Dgw4, we conclude that the dose rates for all samples except these three did not (a)

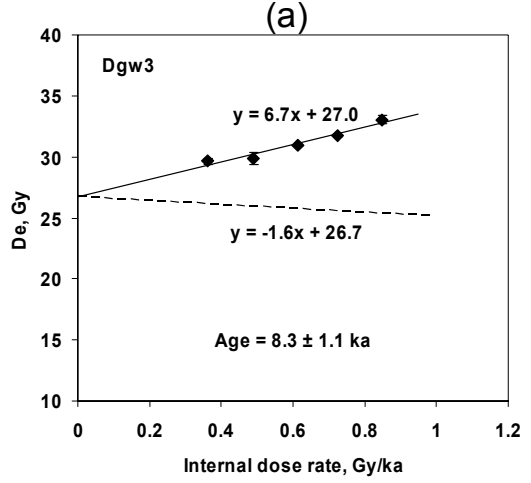

(b)

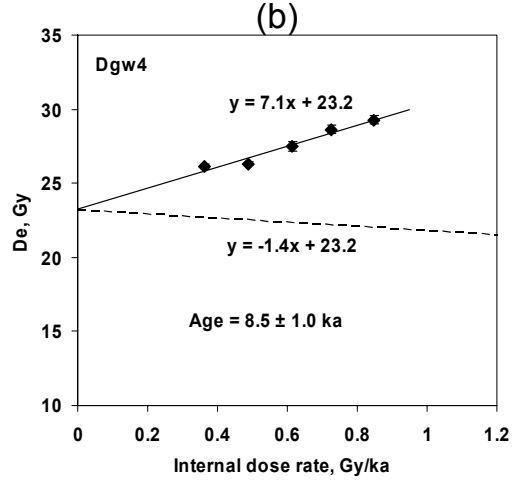

(c)

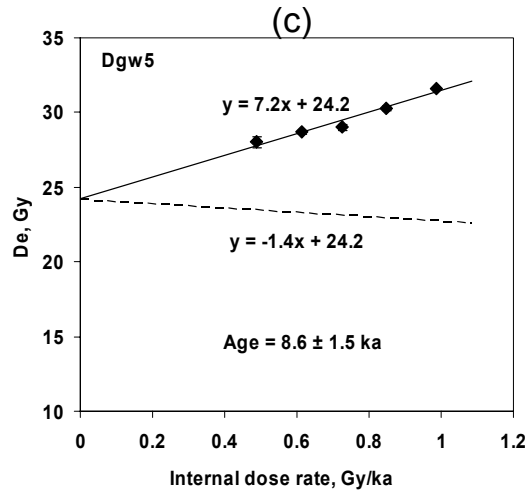

Fig. 4. Results of isochron dating for samples (a) Dgw3; (b) Dgw4; (c) Dgw5. The experimental data for K-feldspars were fitted by a linear function using the least squares method. The dashed line, representing the external dose rate with allowance being made for grain size, has its starting point the extrapolation of the line fitted through the experimental points. 
change significantly after burial; thus the quartz OSL ages for these samples are also reliable.

\section{DISCUSSION}

Since the samples from Dgw1 to Dgw6 show little difference between their $D_{\mathrm{e}}$ values and also have similar ages (from 8 to $9 \mathrm{ka}$ ) deduced from the isochron ages, it would be expected that they should have a similar dose rate. However, for Dgw2, Dgw3 and Dgw4 large variations in dose rates were observed compared to the other samples, indicating that changes in the dose rates for these three samples occurred recently. Since the dose rates measured today are higher than the long-term average for samples Dgw2, Dgw3 and Dgw4, leading to significant underestimation of the quartz OSL age, we conclude that disequilibrium in the uranium or thorium decay series due to the lost of the parent or daughter is not an important influence on the dose rate determination; otherwise it would cause underestimation of the average dose rate rather than overestimation. It also seems unlikely that the $\mathrm{K}$ content has changed significantly since the measured $\mathrm{K}$ contents are similar for all samples throughout the section.

Instead, a reasonable explanation for the age reversal is that significant uptake of radioisotopes, e.g. uranium, occurred recently. Samples from the $40 \mathrm{~cm}$ thick layer at depths of 1.55 to $1.95 \mathrm{~m}$ below the top of the deposits are rich in iron oxide concretions; this increases the possibility of the uptake of radioisotopes, such as uranium (Bruno et al., 1995). To investigate this possibility, the iron oxide concretions were picked out by hand from sample Dgw2 to obtain two fractions, one rich in oxide concretions and the other not. The two fractions were then ground into fine powders and measured using alpha counting. The count rate for the fraction containing the oxide concretions is $21.29 \pm 0.31$ counts $/ \mathrm{ks}$. While the count rate for the other fraction is $16.86 \pm 0.28$ counts $/ \mathrm{ks}$. This indicates that uptake of radioisotopes may be the main reason for overestimation of dose rates in samples Dgw2, Dgw3 and Dgw4.

It is also possible to use the measured isochron $D_{e}$ values together with either the environmental dose rate based on measured $\mathrm{U}$, Th and $\mathrm{K}$ concentrations, or even typical concentrations of these isotopes, e.g. $\mathrm{K}=1.5 \%$, $\mathrm{U}=3 \mathrm{ppm}, \mathrm{Th}=10 \mathrm{ppm}$, cosmic ray dose rate $=0.2 \mathrm{~Gy} / \mathrm{ka}$ and water content $=10 \%$, to determine Fex. This is because the influence of the variation in the environmental dose rate on the isochron age is negligible as it relates to only the relative proportions of the isotopes through the individual values of $\Phi$ for $\mathrm{U}$, Th and $\mathrm{K}$ (see section 2). This can be shown in Table 3, where the isochron ages for Dgw4 and Dgw3 based on measured U, Th and $\mathrm{K}$ concentrations are indistinguishable from those obtained from assumed concentrations. Both sets of isochron ages are consistent with the stratigraphy. This demonstrates the flexibility of the isochron approach as an alternative dating method for samples (such as Dgw2, Dgw3 and Dgw4) for which conventional ages cannot be calculated because of problems with the environmental dose rate.

For overcoming environmental dose rate changes, the isochron method has several advantages over the subtraction method. Firstly, the latter uses measurements of both quartz and feldspar (e.g. Vogel et al., 1999) and thus requires extra sample preparation and measurement time. This was also a problem with the isochron method proposed by Zhao and Li (2002). In addition, the subtraction method is only applicable in cases when the K-feldspar IRSL signal does not fade. In the new isochron method (Li et al., submitted) the internal dose rate is estimated from the estimated $\mathrm{K}$ content of the $\mathrm{K}$-feldspar grains and it has been shown that the IRSL signal resulting from the internal dose rate has not faded (Li et al., 2007). In addition, the isochron method does not rely on the measurement of the quartz OSL signal, that normally shows early saturation with dose, thus restricting the subtraction method to samples typically less than 100 (or even 50) ka.

As applied in this study, one disadvantage is that at least four grain sizes of K-feldspar were measured, which is rather time consuming; however, if two extreme grain size populations are available, e.g. 63-90 $\mu \mathrm{m}$ and $212-250 \mu \mathrm{m}$, a two point isochron line could be used.

\section{CONCLUSIONS}

Significant changes in environmental dose rate were shown to have occurred in a lacustrine and fluvial sediment section in China, with nodules of radioisotopes rich being found in part of the section. Their presence caused significant underestimation of the quartz OSL ages since the latter were calculated using current values of uranium and thorium (as obtained by thick source alpha counting) for the estimation of dose rate. This uptake of radioisotopes appeared to have happened recently. By applying the new isochron method for obtaining ages from measurements made on K-feldspars in a range of grain sizes, it was demonstrated that such changes in dose rate could be overcome and a stratigraphically correct age obtained.

Table 3. Internal dose rate, equivalent doses and ages obtained for K-feldspars (F) and quartz (Q) grains (125-150 $\mu$ m diameter) as well as by the isochron method.

\begin{tabular}{lcccccc}
\hline Sample & $\begin{array}{c}\text { Int. Dose rate } \\
(\mathbf{G y} / \mathbf{k a})\end{array}$ & $\begin{array}{c}\text { De(F) } \\
(\mathbf{G y})\end{array}$ & $\begin{array}{c}\text { Age(F) } \\
(\mathbf{k a})\end{array}$ & $\begin{array}{c}\text { Age(Q) } \\
(\mathbf{k a})\end{array}$ & $\begin{array}{c}\text { Isochron age (a) }^{\mathbf{b}} \text {, } \\
(\mathbf{k a})\end{array}$ & $\begin{array}{c}\text { Isochron age (b) }^{\mathbf{c}}, \\
(\mathbf{k a})\end{array}$ \\
\hline Dgw5 & $0.62 \pm 0.05$ & $28.70 \pm 0.13$ & $8.90 \pm 0.30$ & $8.25 \pm 0.29$ & $8.6 \pm 1.5$ & $8.7 \pm 1.5$ \\
Dgw4 & $0.62 \pm 0.05$ & $27.50 \pm 0.32$ & $6.75 \pm 0.23$ & $6.51 \pm 0.21$ & $8.5 \pm 1.0$ & $8.5 \pm 1.0$ \\
Dgw3 & $0.62 \pm 0.05$ & $30.90 \pm 0.12$ & $5.49 \pm 0.18$ & $4.78 \pm 0.16$ & $8.3 \pm 1.1$ & $8.4 \pm 1.5$ \\
\hline
\end{tabular}

a The internal dose rate is for $\mathrm{K}$-feldspar calculated by assuming $\mathrm{K}=13 \pm 1 \%$ and $\mathrm{Rb}=400 \pm 100 \mathrm{ppm}$.

$\mathrm{b}$ The isochron ages were calculated from measured environmental dose rates.

c The isochron ages were calculated from assumed environmental dose rates given in the text. 
Even without any knowledge of the environmental dose rate, it was shown that it was possible to obtain the isochron age by assuming a typical sedimentary dose rate for the sample. In addition, ages can be obtained irrespective of the past water content, radon loss and changes in cosmic ray dose.

\section{ACKNOWLEDGEMENTS}

The authors thank Dr J.M. Sun for his help in the field work. This study is financially supported by a grant to SHL from the Research Grant Council of the Hong Kong Special Administrative Region, China (Project no. 7106/02P, 7032/05 and 7035/06).

\section{REFERENCES}

Adamiec G and Aitken MJ, 1998. Dose-rate conversion factors: update. Ancient TL 16: 37-50.

Aitken MJ, 1985. Thermoluminescence dating. London, Academic press: $267 \mathrm{pp}$.

Aitken MJ, 1998. An Introduction to Optical Dating. Oxford, Oxford University Press: $359 \mathrm{pp}$.

Auclair M, Lamothe M and Huot S, 2003. Measurement of anomalous fading for feldspar IRSL using SAR. Radiation Measurements 37(4-5): 487-492, DOI 10.1016/S1350-4487(03)00018-0.

Blair MW, Yukihara EG and McKeever SWS, 2005. Experiences with single-aliquot OSL procedures using coarse-grain feldspars. $R a$ diation Measurements 39(4): 361-374, DOI 10.1016/j.radmeas.2004.05.008.

Brennan BJ, 2003. Beta doses to spherical grains. Radiation Measurements 37(4-5): 299-303, DOI 10.1016/S1350-4487(03)00011-8.

Bruno J, Depablo J, Duro L and Figuerola E, 1995. Experimental-Study and Modeling of the U(Vi)-Fe(Oh)(3) Surface Precipitation Coprecipitation Equilibria. Geochimica Et Cosmochimica Acta 59(20): 4113-4123, DOI 10.1016/0016-7037(95)00243-S.

Duller GAT, 2003. Distinguishing quartz and feldspar in single grain luminescence measurements. Radiation Measurements 37(2): 161165, DOI 10.1016/S1350-4487(02)00170-1.

Eriksson MG, Olley JM, Kilham DR, Pietsch T and Wasson RJ, 2006. Aggradation and incision since the very late Pleistoncene in the Naas River, south-eastern Australia. Geomorphology 81(1-2), 6688, DOI 10.1016/j.geomorph.2006.04.001.

Fain J, Soumana S, Montret M, Miallier D, Pilleyre T and Sanzelle S, 1999. Luminescence and ESR dating Beta-dose attenuation for various grain shapes calculated by a Monte-Carlo method. Quaternary Science Reviews 18(2): 231-234, DOI 10.1016/S02773791(98)00056-0.

Huntley DJ, 2002. Comment on "Luminescence Dating of Coastal Sands: Overcoming Changes in Environmental Dose Rate" by J. C. Vogel, A. G. Wintle and S. M. Woodborne. Journal of Archaeological Science 29(5): 559-560, DOI 10.1006/jasc.2001.0721.

Huntley DJ and Baril MR, 1997. The K content of the K-feldspars being measured in optical dating or in thermoluminescence dating. Ancient TL 15: 11-13.

Huntley DJ and Hancock RGV, 2001. The Rb contents of the Kfeldspars being measured in optical dating. Ancient TL 19: 43-46.

Huntley DJ and Lamothe M, 2001. Ubiquity of anomalous fading in Kfeldspars and the measurement and correction for it in optical dating. Canadian Journal of Earth Sciences 38(7): 1093-1106, DOI 10.1139/cjes-38-7-1093.

Huot S and Lamothe M, 2003. Variability of infrared stimulated luminescence properties from fractured feldspar grains. Radiation Measurements 37(4-5): 499-503, DOI 10.1016/S13504487(03)00014-3.

Li S-H, 1994. Optical dating: insufficiently bleached sediments. Radiation Measurements 23(2-3), 563-567, DOI 10.1016/13504487(94)90100-7.

Li S-H, 2001. Identification of well-bleached grains in the optical dating of quartz. Quaternary Science Reviews 20(12): 1365-1370, DOI 10.1016/S0277-3791(00)00156-6.

Li S-H, Sun JM and Zhao H, 2002. Optical dating of dune sands in the northeastern deserts of China. Palaeogeography Palaeoclimatology Palaeoecology 181(4): 419-429, DOI 10.1016/S00310182(01)00443-6.

Li B, Li S-H, Wintle AG and Zhao H, 2007. Isochron measurements of naturally irradiated K-feldspar grains. Radiation Measurements 42(8): 1315-1327, DOI 10.1016/j.radmeas.2007.09.008.

Li B, Li S-H, Wintle AG and Zhao, H., Isochron dating of sediments using K-feldspars. Journal of Geophysical Research (submitted).

Marley NA, Gaffney JS, Orlandini KA and Cunningham MM, 1993. Evidence for Radionuclide Transport and Mobilization in a Shallow, Sandy Aquifer. Environmental Science \& Technology 27(12): 2456-2461, DOI 10.1021/es00048a022.

McKerrell HV and Mejdahl V, 1981. Progress and problems with automated TL dating, Proceedings of the 16th International Symposium of Archaeometry, National Museum of Antiquities of Scotland, Edingurgh: pp. 36.

Mejdahl V, 1979. Thermoluminescence Dating: Beta-Dose Attenuation in Quartz Grains. Archaeometry 21(1): 61-72, DOI 10.1111/j.1475-4754.1979.tb00241.x.

Mejdahl V, 1983. Feldspar inclusion dating of ceramics and burnt stones. PACT 9: 351-364

Mercier N, Valladas H, Joron JL, Schiegl S, Bar-Yosef O and Weiner S, 1995. Thermoluminescence Dating and the Problem of Geochemical Evolution of Sediments - a Case-Study - the Mousterian Levels at Hayonim. Israel Journal of Chemistry 35(2): 137-141.

Munyikwa K, 2000. Cosmic ray contribution to environmental dose rates with varying overburden thickness. Ancient TL 18: 27-34.

Murray AS and Wintle AG, 2000. Luminescence dating of quartz using an improved single-aliquot regenerative-dose protocol. Radiation Measurements 32(1): 57-73, DOI 10.1016/S1350-4487(99)00253$\mathrm{X}$.

Olley JM, Murray A and Roberts RG, 1996. The effects of disequilibria in the uranium and thorium decay chains on burial dose rates in fluvial sediments. Quaternary Science Reviews 15(7): 751-760, DOI 10.1016/0277-3791(96)00026-1.

Olley JM, Roberts RG and Murray AS, 1997. Disequilibria in the uranium decay series in sedimentary deposits at Allen's Cave, Nullarbor Plain, Australia: Implications for dose rate determinations. Radiation Measurements 27(2): 433-443, DOI 10.1016/S13504487(96)00114-X.

Prescott JR and Hutton JT, 1994. Cosmic-Ray Contributions to DoseRates for Luminescence and Esr Dating - Large Depths and LongTerm Time Variations. Radiation Measurements 23(2-3): 497-500, DOI 10.1016/1350-4487(94)90086-8.

Sun JM, 2000. Origin of eolian sand mobilization during the past 2300 years in the Mu Us Desert, China. Quaternary Research 53(1): 7888, DOI 10.1006/qres.1999.2105.

Vogel JC, Wintle AG and Woodborne SM, 1999. FOCUS: Luminescence dating of coastal sands: Overcoming changes in environmental dose rate. Journal of Archaeological Science 26(7): 729. 733, DOI 10.1006/jasc.1999.0450.

Wallinga J, Murray AS and Wintle AG, 2000. The single-aliquot regenerative-dose (SAR) protocol applied to coarse-grain feldspar. $R a$ diation Measurements 32(5-6): 529-533, DOI 10.1016/S13504487(00)00091-3.

Wintle AG, 1973. Anomalous Fading of Thermoluminescence in Mineral Samples. Nature 245(5421): 143-144, DOI 10.1038/245143a0.

Wintle AG, 1997. Luminescence dating: Laboratory procedures and protocols. Radiation Measurements 27(5-6): 769-817, DOI 10.1016/S1350-4487(97)00220-5.

Zhang J-F, Li S-H and Tso MYW, 2001. Improvement of the equivalent dose determination using aliquots of potassium feldspars. Radiation Measurements 33(1): 65-71, DOI 10.1016/S13504487(00)00132-3.

Zhao $\mathrm{H}$ and $\mathrm{Li} \mathrm{S}-\mathrm{H}, 2002$. Luminescence isochron dating: A new approach using different grain sizes. Radiation Protection Dosimetry 101(1-4): 333-338.

Zhao $\mathrm{H}$ and $\mathrm{Li} \mathrm{S}-\mathrm{H}, 2005$. Internal dose rate to $\mathrm{K}$-feldspar grains from radioactive elements other than potassium. Radiation Measurements 40(1): 84-93, DOI 10.1016/j.radmeas.2004.11.004. 